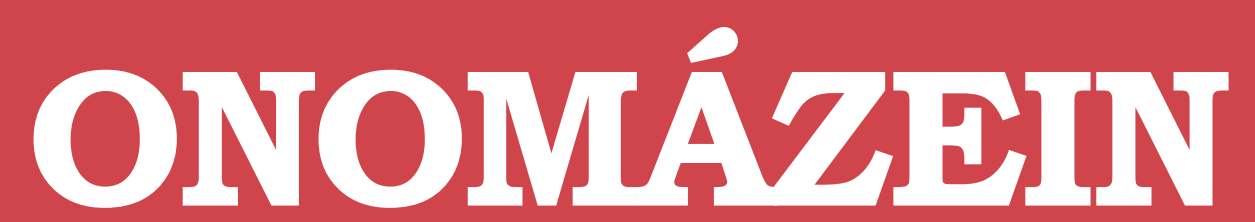

Revista semestral de lingüística, filología y traducción
PONTIFICIA UNIVERSIDAD

\title{
Análisis de algunos componentes textuales del proyecto de tesis doctoral en Educación: título, justificación del problema y recurso a otros textos
}

Analysis of some textual components of the project for doctoral thesis in Education: title, justification of the problem and recourse to other texts

\section{Hilda Difabio de Anglat}

Universidad Nacional de Cuyo

Argentina

\section{(C) $\underset{\mathrm{BY}}{(\mathrm{i})}$}

Hilda Difabio de Anglat: Facultad de Filosofía y Letras, Universidad Nacional de Cuyo; Centro de Investigaciones de Cuyo, Consejo Nacional de Investigaciones Científicas y Técnicas, Argentina.

Correo electrónico: ganglat@gmail.com 


\section{Resumen}

El proyecto de tesis doctoral es un texto de importancia en tanto garantía de que las decisiones respecto de la fase teórica, la formulación de los objetivos, los métodos propuestos, estén debidamente alineadas con las prácticas de la buena investigación. sin embargo, no es un género explícitamente bajo examen, escrutinio inexistente en el caso de propuestas de formalidad pedagógica. En este trabajo analizamos, desde encuadres teóricos y procedimientos analíticos probados, 31 proyectos de dos doctorados en Educación de la Universidad Nacional de Cuyo y de la Universidad Católica de Cuyo, Argentina, con el objeto de explorar qué clases de títulos aparecen, cómo se justifica el problema y qué modalidades adopta la incorporación de otros textos (citación y referenciación). Se seleccionan estos componentes textuales porque son los que se asocian directamente con la lógica inicial de una investigación, cuya relevancia reside en que es el requisito sine qua non que debe satisfacer un proyecto para ser aprobado. Los resultados muestran que las clases de títulos configuran un conjunto restringido en el que prevalece la construcción nominal que anuncia el tema; la justificación del problema aparece como un área débil, con preeminencia de la basada en su importancia y en el aporte de los hallazgos; prima la función atributiva de la citación, esto es, la cita asociada a un uso enciclopédico de las publicaciones referidas.

Palabras clave: proyecto de investigación; doctorado en Educación; título del proyecto; justificación del problema; citación y referenciación.

\section{Abstract}

The PhD project is a text of importance in ensuring that decisions regarding theoretical stage, the goal formulation, proposed methods, are duly aligned with practices of good research. However, it is not a genre explicitly under examination, non-existent scrutiny in the case of proposals of pedagogical formality. In this paper we analyze, from theoretical frames and analytical procedures proven, 31 projects from two doctorates in Education of the National University of Cuyo and of the Catholic University of Cuyo, Argentina, in order to explore what kinds of titles show up, how problem is warranted and which modalities adopts the incorporation of other texts (citation and referencing). These textual components are selected because they are directly associated with the initial logic of an inquiry, whose relevance resides in that it is the requirement sine qua non that must satisfy a project to be approved. The results show that types of titles form a restricted set wherein prevails the nominal construction that announces the subject; the justification of problem appears as a weak area with preeminence of the ones based on its importance and on the contribution of the findings; predominates the attributive function of the citation, i.e., citation associated to an encyclopedic use of the referred publications.

Keywords: research project; doctorate in Education; project title; justification of the problem; citation and referencing. 


\section{Introducción}

En el ámbito de la comunicación científica no hay riesgo de ambigüedad respecto de los géneros discursivos por cuanto representan registros especializados característicos de una comunidad sociorretórica, la que establece una "correspondencia inequívoca entre los aspectos textuales y la identidad que otorga un nombre (i.e. 'tesis', 'monografía', 'comunicación', etc.)" (Bermúndez, 2008: 7).

El proyecto, del latín proiectus (idear, dar forma, forjar, elaborar), refiere a un texto cuya finalidad es exponer una tarea que se piensa llevar a cabo; es "la previsión de los medios adecuados para lograr fines futuros" (Paredes de Meaños, 1997: 3). Para que la idea planteada se pueda transformar en una alternativa viable, prevé no sólo lo que se piensa hacer sino cómo hacerlo.

En nuestro análisis, nos ocuparemos de los componentes directamente implicados en plasmar la lógica inicial del estudio - el título, la justificación del problema por investigar y el recurso a otros textos-, cuya relevancia reside en que es el requisito sine qua non que debe satisfacer la propuesta para ser aprobada.

Los objetivos específicos de esta presentación son: a) identificar qué clases de títulos se distinguen en un corpus de proyectos de investigación de nivel doctoral, b) ponderar cómo se justifica la investigación y c) indagar qué modalidades asume la incorporación de otros textos.

Aunque el proyecto reviste una importancia central -en tanto garantía de que las decisiones respecto de la fase conceptual, la formulación de los objetivos, los métodos propuestos, sean debidamente alineadas con las prácticas de la buena investigación-, no es un tipo de texto explícitamente en estudio; en este sentido, hasta donde hemos podido indagar, no hay análisis previos de la propuesta de tesis doctoral de formalidad educativa. Por ello, nuestro propósi- to es realizar un acercamiento a este género discursivo, con la intención última de asistir a los alumnos en su elaboración.

\section{Los referentes teóricos}

\subsection{El título}

En su función designativa, el título de un texto científico —-definido como "el menor número de palabras que describen adecuadamente su contenido" (Day, 1990: 15) - es, sin duda alguna, un componente privilegiado del discurso académico en tanto tarjeta de presentación del trabajo (Jara, 1999). En su génesis psicognoseológica, debe ser el producto de una generalización constructiva (Piaget, 1984), la que concibe una organización inédita, una integración de rango superior de diferenciaciones y coordinaciones; de allí su originalidad y claridad. En su formulación psicolingüística, ha de ser el resultado de un proceso de síntesis en una sola macroproposición (Van Dijk y Kintsch, 1983); de allí la exigencia de concisión.

No obstante su importancia, es una dimensión de los géneros académicos que no ha sido estudiada en profundidad (Soler, 2007). De hecho, el relevamiento bibliográfico destaca una única categorización reciente de títulos (de artículos científicos): la realizada por Hartley (2007, 2008), que actualiza y extiende un trabajo temprano (Crosby, 1976). En esta taxonomía distinguimos ocho tipos - subsumiendo en el octavo categorías que el autor separa- según que el título: 1) enuncie el tema general (ej.: La escritura de artículos científicos en Inglés $\left.{ }^{1}\right) ; 2$ particularice el tema luego del título general (ej.: El rol de los valores en la investigación educativa: el lugar de la reflexión); 3) formule la pregunta de investigación (ej.: ¿En qué consiste la práctica basada en la evidencia?; 4) indique que la respuesta a la pregunta será revelada (ej.: Resultados actuales de la investigación sobre la estructuración del abstract); 5) anticipe la tesis, la dirección del ar- 
gumento del autor (ej.: El perdido arte de la conversación); 6) enfatice la metodología empleada (ej.: El uso de subtítulos: una revisión meta-analítica); 7) sugiera directrices o cursos de acción (ej.: Diecinueve estrategias para obtener un doctorado); 8) promueva la atención — mediante el empleo de una apertura sorprendente, aliteración, alusión literaria o bíblica, juego de palabras o proposición que desconcierta- (ej.: Corriendo el velo de la defensa de la tesis: las experiencias de estudiantes graduados).

\subsection{Justificación del problema por investigar}

Establecido el espacio vacante (Swales, 1990), el problema no investigado todavía, se requiere fundamentar la propuesta de tesis, esto es, desarrollar la argumentación que la avala, suministrando una exposición convincente sobre las razones de su valor y la relevancia de la acción por realizar. Este apartado debe, entonces, explicar en qué grado y de qué modo la investigación es importante para el avance potencial de la disciplina en la cual el doctorando encuadra su propuesta. El problema ha de ser significativo para que se justifique su investigación, para que aparezca como "valiendo la pena" el tiempo y el esfuerzo que demandará.

Por su exhaustividad y potencia heurística (ya que proporciona una definición operativa de cada categoría), se aplica la categorización de Sabaj Meruane y Landea Balin (2012) en siete tipos de justificación: 1) falta genérica de conocimiento; 2) falta de conocimiento práctico; 3) basada en la importancia del tema; 4) basada en los aportes de los hallazgos; 5) basada en un vacío metodológico; 6) basada en la solución de un problema; 7) basada en la corroboración de una teoría.

\subsection{Citación y referenciación}

Dado que la ciencia contemporánea se basa en el conocimiento acumulado, en la escritura académica el recurso a otros textos no representa una estrategia posible sino un componente indispensable y prototípico (Jakobs, 2003). Cada "préstamo" de dicha literatura científica debe estar claramente identificado como tal por el acto lingüístico de citación - acompañada por la referencia bibliográfica de la fuente (Romera Iruela, 1996) - con un propósito bien definido (Reyes, 1999; Jakobs, 2003; Massi, 2005): integrar la producción del autor en el conocimiento compartido, recuperar la voz experta en el tema que se investiga, ilustrar lo que se está diciendo, apoyar y justificar enfoques, evaluar críticamente las diferentes posiciones.

Un proyecto de investigación debe informar qué se sabe sobre el tema y en qué contexto epistemológico se va a insertar la pregunta que lo guía. En este sentido, señalan Boote y Beile (2005: 6) -en un artículo titulado Scholars before Researchers (Académicos antes que Investigadores)_: "Una revisión sustancial, completa, cuidadosa de la literatura es una condición para realizar una investigación sustancial, completa, cuidadosa. La 'buena' investigación es buena porque incrementa nuestra comprensión colectiva. (...) Shulman utiliza el término generatividad (...) [para referirse] a la habilidad de edificar desde los que nos precedieron." Empleando una imagen de Saltalamacchia (2005), el conocimiento acumulado cumplirá la función del peldaño en el que estamos parados en el momento de subir la escalera: precede y sirve de apoyo para el esfuerzo que habrá de conducir a un escalón más elevado. Por ello, el doctorando debe realizar "los trabajos preparatorios necesarios para asegurarse de que esta investigación completará y no duplicará los esfuerzos de otros" (Wainerman, 1998: 26).

La naturaleza compleja y desordenada de los problemas en Educación hace que la generatividad en esta área sea más difícil que en otras disciplinas y demanda que desarrollemos revisiones muy cuidadosas de la literatura.

Dichas revisiones quedan de manifiesto en las citas que se incorporan al texto: integrales (o integradas) y no integrales o (no integradas) — según la categorización de Swales (1990)—, literales o no literales (Reyes, 1999; Sabaj Meruane 
y Páez Muñoz, 2011) o estilo directo e indirecto (Massi, 2005).

En la cita integrada, la fuente a que refiere cumple una función sintáctica en el enunciado (Swales, 1990), como sujeto, agente o adjunto reportativo (Sabaj Meruane y Páez Muñoz, 2011); la no integrada, en cambio, carece de dicha función en tanto sólo menciona entre paréntesis el apellido del autor y el año de la publicación (siguiendo las normas APA —American Psychological Association-, que son las recomendadas para los proyectos en examen). Se produce, entonces, una diferencia de énfasis: en el autor como enunciador autorizado de un contenido vs. en el contenido.

La cita literal es un fragmento de otro texto reproducido al pie de la letra, lo que se indica mediante comillas inglesas si son breves o mediante letra bastardilla si tienen más de cinco líneas, en cuyo caso se separan del texto entre márgenes y a un interlineado menor; además de los datos de autor y año, incluye la referencia al número de página de la cual se extrae. Es un procedimiento de estilo directo por su literalidad o repetición verbatim del discurso de origen (Massi, 2005).

En la cita no literal, la intención predominante del emisor no es trasmitir la forma del discurso fuente sino sólo su contenido (Dúo de Brottier, 2005); luego, presenta una paráfrasis, síntesis o comentario de las ideas de otro autor y se puede reconocer pues aparece mencionado junto con el año de la publicación (Sabaj Meruane y Páez Muñoz, 2011). Representa un estilo indirecto a partir de la modificación de algunas marcas enunciativas, sin alterar el contenido (Massi, 2005).

La combinación de ambos criterios da lugar a cuatro categorías: 1) integrada literal; 2) integrada no literal; 3) no integrada literal y 4) no integrada no literal.

Por otra parte, "la cita establece un tipo específico de relación (cumple una función) respecto del texto madre" (Sabaj Meruane y Páez Muñoz,
2011: 118-119). En nuestro estudio, por su operatividad para analizar los proyectos de escritores mayoritariamente no expertos, aplicamos la clasificación de Sánchez (2011), quien a su vez la toma adaptada de Petrić (2007) y, como consecuencia, de Thompson (2001), modelo en que se basa dicha investigadora. Las categorías de Sánchez son diez, pero desestimamos "Comparación de los resultados propios con otras fuentes", ya que este tipo de cita se justifica cuando se discuten los productos del análisis de los datos (Petrić, 2007). A continuación, se caracteriza brevemente cada una de las funciones:

Atribución: Refiere a la fuente de donde proviene la información; atribuye la proposición a un otro (Thompson y Tribble, 2001), lo que confiere a la cita un valor informativo; "reconoce implícitamente una deuda con el trabajo de un autor precedente” (Sánchez, 2013: 87).

Ejemplificación: Aporta información útil para ilustrar una proposición, que opera como evidencia específica de apoyo y refuerzo del argumento. Suele ir precedida de marcadores del discurso ("por ejemplo", "como ejemplo", "a saber", "para ilustrar", etc.) que facilitan su identificación en el texto. En las citas no integrales, es frecuente que se relegue esta información a la nota al pie de página, en tanto el escritor considera que interrumpe la progresión temática.

Referencia: Redirige al lector a otro/s trabajo/s - por lo general, subsidiarios-, con la finalidad de que aquel pueda ampliar la información sobre el asunto. Suele aparecer como cita no integral entre paréntesis, viene precedida normalmente por abreviaturas ("vid.", "cfr.") o formas verbales ("ver" o "véase").

Declaración de uso: Señala que se emplea el trabajo citado o una de sus partes (por ejemplo, instrumento/s) en la propuesta; con frecuencia, explica el propósito de su empleo.

Aplicación: Establece conexiones con la pu- 
blicación citada a fin de aprovechar los argumentos, los conceptos, la terminología o los procedimientos, desde los objetivos propios (Petrić, 2007).

La delimitación de las fronteras entre declaración de uso y aplicación "no está del todo clara y puede generar problemas en la decisión de clasificar las citas bajo una u otra categoría” (Sánchez, 2013: 89).

Evaluación: Por lo general, pondera el estudio referenciado en sus méritos, si bien pueden aparecer críticas negativas, las que suelen incluir una evaluación positiva previa. También, puede poner en duda una afirmación sin cuestionarla abiertamente; este desacuerdo de opinión es "una evaluación del trabajo citado, aunque no pueda considerarse como una crítica negativa de forma explícita” (Sánchez, 2013: 90).

Enlace entre fuentes: Señala vínculos o contrastes entre los textos; en el primer caso, para otorgar fuerza al argumento o para definir un concepto; en el segundo, para mostrar los diferentes puntos de vista respecto del tema.

Concurrencia ${ }^{2}$ : Concentra citas de varios autores en torno de una afirmación, a fin de subrayar su relevancia en tanto se considera conocimiento general en el campo que se está investigando. Sirve también para demostrar el dominio del tema, pues prueba la lectura exhaustiva de la bibliografía disponible sobre ese asunto. Se diferencia de la atribución en el énfasis y en la intensidad que significan el refuerzo de una afirmación por la autoridad de varios expertos.

Autocita: Referencia en la que el autor se cita a sí mismo, "con el fin de mostrar un conocimiento acumulado sobre un tema $y$, por lo tanto, su dominio sobre el asunto que describe" (Sánchez, 2011: 188). Si bien puede cumplir cualquiera de las funciones retóricas anteriormente definidas, se considera oportuno separarla (como hace el referido autor) porque informa sobre la preparación previa en investigación, uno de los factores que inciden en la eficacia terminal del doctorado (entre otros, D’Andrea, 2002; Carlino, 2005; Difabio de Anglat, 2011).

\section{Metodología}

\subsection{Propósito y preguntas de la investigación}

Como señaláramos, nuestro propósito es analizar proyectos de tesis doctoral en Educación a fin de dar respuesta a tres preguntas asociadas con la lógica inicial de una investigación:

- ¿Qué clases de títulos se pueden distinguir en el corpus?

- ¿Cómo se justifica la investigación?

- ¿Qué modalidades asume la incorporación de otros textos?

\subsection{El corpus}

Del total de 65 proyectos aprobados por los comités académicos del Doctorado en Ciencias de la Educación, Universidad Nacional de Cuyo (Mendoza, Argentina) y del Doctorado en Educación, Universidad Católica de Cuyo (San Juan, Argentina) entre setiembre de 2008 y setiembre de 2012 , el corpus se constituye de 31 proyectos escritos en español como primera lengua3, que nos fueran remitidos por los propios doctorandos; esto es, si bien teníamos acceso a las propuestas como miembro de los respectivos comités académicos, las solicitamos vía correo electrónico a los alumnos a modo de consentimiento informado y sólo incorporamos al corpus las correspondientes a quienes respondieron a la convo-

2 Hemos preferido designar esta categoría como "concurrencia” en lugar de "competencia”, el nombre que le asigna Sánchez en seguimiento de Petrić (2007).

3 Excluimos del número total de proyectos y de la convocatoria a los estudiantes brasileños que cursan el doctorado en la Universidad Nacional de Cuyo. 
catoria. Los 31 textos sumaron 265.159 palabras en total.

\subsection{Procedimientos de análisis}

El análisis se realizó manualmente -con apoyo de las herramientas del programa Worddesde una perspectiva cualitativa y cuantitativa, en ese orden. En los tres componentes textuales, aplicamos encuadres teóricos probados, esto es, categorías "prestadas" (en la denominación de Bertely Busquets, 2000) o "códigos in-vitro" (Glaser y Strauss, 1967). Respecto del título, para una mayor discriminación, fue necesario elaborar también categorías nuevas en tanto procedentes del conjunto de conceptos descubiertos en los datos (categorías "propias" o "códigos in vivo"). Desde la lingüística del corpus, prima entonces el enfoque basado en el corpus en tanto se lo utiliza para ejemplificar categorías predeterminadas (Parodi, 2005), el que se combina en el último caso con el enfoque guiado por el corpus puesto que algunas categorías son "el producto de la evidencia resultante del análisis" (Beke, 2008: 18).

En relación con la justificación de la propuesta, se comenzó el análisis en el apartado Formulación y fundamentación del problema por investigar ${ }^{4}$, porque es evidente que corresponde a "aquel extracto textual, de carácter argumentativo, donde el autor da cuenta de la necesidad o las razones de ser de la investigación” (Sabaj Meruane y Landea Balin, 2012: 325), para luego considerar la totalidad del proyecto a fin de identificar si aparecían nuevas justificaciones en otros apartados.

Con respecto a la citación, se empleó como criterio de reconocimiento de la cita la atribución del mensaje a un autor específico. Como en Beke (2008), se desestimaron las citas y referencias que aluden a documentos (por ejemplo, Ley Federal de Educación, Ley de Educación Nacional), puesto que son un producto colectivo sin particularización manifiesta de los integrantes del grupo y, en este sentido, no se explicitan los nombres.

Mediante la función "control de cambios" del programa Word se generó un comentario para cada una, comenzando por la clasificación en integrada y no integrada, literal y no literal. Este análisis resultó sencillo por la presencia de marcadores específicos (el lugar del autor en el texto o la referencialidad - autor/a, investigador/a, aquel/la, él/ella-, comillas inglesas o letra bastardilla). La codificación según la función retórica de la cita fue mucho más laboriosa: adscribimos cada comentario a una categoría mediante el método del análisis funcional de las citas (Thompson, 2001; Petrić, 2007; Sánchez, 2011) desde el contexto - verbos introductorios, cotexto lingüístico-y desde el examen de su contenido conceptual.

Además, calculamos la cantidad de publicaciones incluidas en la sección Referencias o Bibliografía, distinguiendo el tipo de publicación: libro, capítulo de libro, artículo científico y otros (ponencia en evento científico, tesis, diccionario y páginas web).

Dado que el análisis de un corpus tan extenso, sobre todo para determinar las funciones de las citas, es un proceso que demanda mucho tiempo como para solicitar validación inter-juez, adoptamos —como en Sánchez (2012) - el procedimiento viable: reanálisis de cada proyecto, con un período de separación de dos meses, y nueva ponderación de aquellos casos en los que no logramos correspondencia, por su contraste con la tipología y con la ejemplificación de los respectivos autores, hasta arribar a una decisión firme.

En su faz cuantitativa, el estudio se centró en calcular estadísticos descriptivos (frecuencia absoluta y porcentaje, media, mediana, desviación estándar - D. S. - y amplitud intercuartil) y dos pruebas de inferencia estadística (Chi² y diferencia de proporciones). 


\section{Resultados y discusión}

\subsection{El título}

De la tipología de Hartley $(2007,2008)$ sólo pudimos asumir tres categorías (Anuncio del tema general, Particularización del tema luego del título general y Énfasis en la metodología empleada en la investigación luego del título general); por otra parte, el análisis mostró la necesidad de agregar otras tres emergentes de los datos, como subtipos de la primera, en tanto varios títulos de este conjunto de propuestas se distinguían por la indicación expresa de la modalidad del estudio (cfr. tabla 1 ).

En la tabla 2 y en el gráfico I se muestra la cantidad de títulos de los proyectos en cada categoría.

Tal como exhibe el gráfico I, la categoría anuncio del tema general con sus subcategorías representa el $61 \%$ de los títulos del corpus; la diferencia no resulta estadísticamente significa-

\section{TABLA 1}

Clases de títulos en el corpus

\begin{tabular}{|c|c|}
\hline Tipos de títulos & Ejemplo \\
\hline Anuncio del tema general & Modelo pedagógico-didáctico de valoración perfectiva \\
\hline $\begin{array}{l}\text { - como relación entre } \\
\text { variables o categorías }\end{array}$ & $\begin{array}{l}\text { Hacia un modelo multidimensional de la motivación orientada al futuro en } \\
\text { relación con el aprendizaje autorregulado en estudiantes universitarios }\end{array}$ \\
\hline - de alcance explicativo & $\begin{array}{l}\text { Incidencia de la didáctica personalizada en el desarrollo del } \\
\text { aprendizaje autorregulado en estudiantes universitarios }\end{array}$ \\
\hline - de tesis de autor & $\begin{array}{l}\text { Proyecto educativo y medios de comunicación social } \\
\text { desde el pensamiento de Zygmunt Bauman5 }\end{array}$ \\
\hline $\begin{array}{l}\text { Particularización del tema } \\
\text { luego del título general }\end{array}$ & $\begin{array}{l}\text { La enseñanza de la variación lingüística en el nivel medio a partir de la Ley } \\
\text { de Educación Nacional: lineamientos curriculares y propuestas editoriales }\end{array}$ \\
\hline $\begin{array}{c}\text { Énfasis en la metodología } \\
\text { empleada en la investigación } \\
\text { luego del título general }\end{array}$ & $\begin{array}{l}\text { La evaluación didáctica desde las representaciones sociales de los docentes: un } \\
\text { análisis de caso en una unidad académica de la Universidad Nacional de Cuyo }\end{array}$ \\
\hline
\end{tabular}

\section{TABLA 2}

Frecuencia y porcentaje de clases de títulos en el corpus

\begin{tabular}{l|c|c}
\multicolumn{1}{c|}{ Tipos de títulos } & Frecuencia & Porcentaje \\
\hline Anuncio del tema general & 9 & $29 \%$ \\
\hline - como relación entre variables o categorías & 4 & $13 \%$ \\
\hline - de alcance explicativo & 4 & $13 \%$ \\
\hline - de tesis de autor & 2 & $6 \%$ \\
\hline Particularización del tema luego del título general & 8 & $26 \%$ \\
\hline Énfasis en la metodología empleada luego del título general & 4 & $13 \%$ \\
\hline \multicolumn{1}{|c|}{ Total } & 31 & $100 \%$ \\
\hline
\end{tabular}

5 Si bien este título podría adscribirse también a "relación entre variables o categorías", su núcleo diferencial es el abordaje de la asociación planteada desde la obra de un autor. 


\section{GRÁFICO 1}

Distribución porcentual de las clases de títulos

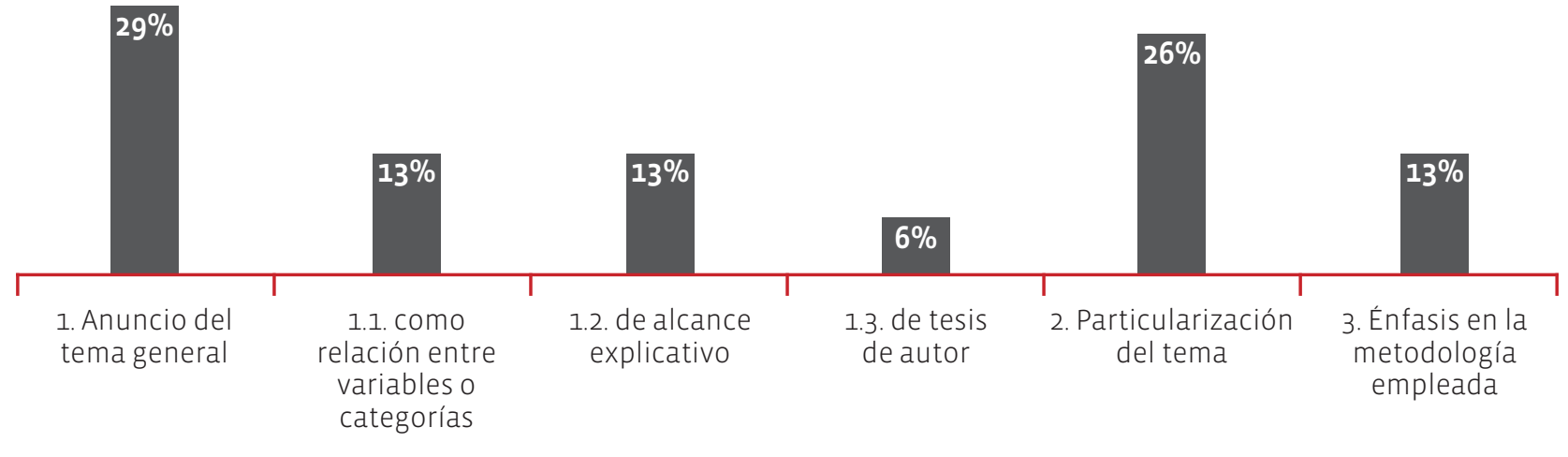

tiva $(z=1,83)^{6}$ respecto de particularización del tema general, pero sí en relación con énfasis en la metodología empleada $(z=2,38)$.

\subsection{Justificación del problema por investigar}

Se halla que tres doctorandos no aportan justificación alguna en tanto, en el apartado correspondiente, sólo anticipan juicios descriptivos no valorativos? ${ }^{7}$. Los restantes presentan desde una sola a un máximo de cuatro (cfr. tabla 3).

En lasiguientetabla, seilustracadacategoría:

\begin{tabular}{|c|c|c|}
\hline & Frecuencia & Porcentaje \\
\hline Ninguna & 3 & 9,7 \\
\hline Una & 7 & 22,6 \\
\hline Dos & 13 & 41,9 \\
\hline Tres & 4 & 12,9 \\
\hline Cuatro & 4 & 12,9 \\
\hline Total & 31 & 100 \\
\hline
\end{tabular}

\section{TABLA 4}

Ejemplificación de los tipos de justificación extraída del corpus

\begin{tabular}{c|c}
\hline \multicolumn{1}{c|}{ Tipo de justificación } & \multicolumn{1}{c}{ Ejemplo } \\
\hline \multirow{2}{*}{ Falta genérica de conocimiento } & $\begin{array}{l}\text { "Esta tesis doctoral tendrá la particularidad de investigar acerca de la enseñanza } \\
\text { filosófica en el nivel primario desde una perspectiva realista con el fin de alcanzar } \\
\text { una educación intelectual integral. De esta manera, el punto de partida es } \\
\text { totalmente distinto de lo realizado hasta ahora si tenemos en cuenta que los } \\
\text { autores del movimiento 'aprender a pensar' se enmarcan en el idealismo". }\end{array}$ \\
\hline \multirow{2}{*}{ Falta de conocimiento práctico } & $\begin{array}{l}\text { "Si bien son variadas las investigaciones que se ocupan de las concepciones } \\
\text { de aprendizaje de los estudiantes en diferentes dominios, estos estudios aún } \\
\text { no avanzan sobre las acciones necesarias para que progresivamente estas } \\
\text { concepciones puedan ir cambiando en pos de aprendizajes más constructivos". }\end{array}$ \\
\hline
\end{tabular}

6 El valor crítico que habría que rebasar para la significatividad estadística en la comparación de proporciones es z = 1,96 a un nivel de confianza $\alpha=0,05$.

7 Categoría que extraemos de nuestra jerarquía de modalidades de razonamiento práctico (Difabio de Anglat, 2005) desde "juicio reductivo" a "juicio crítico-valorativo", la que ubica a esta modalidad del juicio en segundo lugar porque representa una extrapolación de los procesos propios del pensamiento especulativo que es insuficiente en el área de la estimación particular. 


\begin{tabular}{|c|c|}
\hline $\begin{array}{l}\text { Basada en la importancia } \\
\text { del tema }\end{array}$ & $\begin{array}{l}\text { "Se trata de una cuestión de importancia ya que los paradigmas dicen a los } \\
\text { profesionales en Ciencias de la Educación (...) qué hacer sin necesidad de largas } \\
\text { consideraciones existenciales o epistemológicas. Por ello, buscamos analizar } \\
\text { críticamente los problemas epistemológicos actuales (pluralismo, relativismo, } \\
\text { pragmatismo, falta de coherencia entre ontología-epistemología-metodología de } \\
\text { las propuestas educacionales)". }\end{array}$ \\
\hline $\begin{array}{l}\text { Basada en los aportes } \\
\text { de los hallazgos }\end{array}$ & $\begin{array}{l}\text { "El interés central radica en que el trabajo pueda constituirse en un punto } \\
\text { de referencia para los docentes, con el fin de evitar que operen como meros } \\
\text { ejecutores de propuestas externas y, especialmente, de concientizarlos sobre la } \\
\text { importancia de examinar los marcos teóricos necesarios para reflexionar sobre } \\
\text { sus prácticas y sustentarlas de forma consistente. Asimismo, puede contribuir } \\
\text { con los investigadores del campo de la educación y con los autores y editores de } \\
\text { textos escolares, al momento de evaluar, revisar y/o perfeccionar producciones } \\
\text { editoriales que atañen a la enseñanza de contenidos propios de una determinada } \\
\text { área disciplinar". }\end{array}$ \\
\hline $\begin{array}{l}\text { Basada en un vacío } \\
\text { metodológico }\end{array}$ & $\begin{array}{l}\text { "Los recursos metodológicos por emplear podrán ser aplicados en caso de } \\
\text { experiencias que se efectúen en contextos similares, tanto respecto de los nuevos } \\
\text { instrumentos que surgirán como resultado de la relación dialéctica que se propone } \\
\text { lograr entre los participantes de una propuesta de investigación-acción, como de } \\
\text { la utilización de instrumentos existentes con un enfoque innovador nacido de las } \\
\text { interrelaciones derivadas de este estudio". }\end{array}$ \\
\hline $\begin{array}{l}\text { Basada en la solución } \\
\text { de un problema }\end{array}$ & $\begin{array}{l}\text { "El tema seleccionado se presenta de particular interés a partir de la necesidad } \\
\text { de producir cambios y una mejora sustantiva en los resultados referidos a las } \\
\text { habilidades propias de la producción del texto escrito. En este sentido, los } \\
\text { operativos de evaluación de orden nacional (...) arrojan resultados descendidos en } \\
\text { este tema particular y los datos obtenidos no muestran evolución significativa en } \\
\text { cada operativo realizado. Las diferencias de calidad tampoco parecen atribuibles } \\
\text { a los contextos socioeducativos, ni socioeconómicos. En principio, estos factores } \\
\text { habilitarían la idea de que nos encontramos frente a un problema estrictamente } \\
\text { de enseñanza (...)". }\end{array}$ \\
\hline $\begin{array}{l}\text { Basada en la corroboración } \\
\text { de una teoría }\end{array}$ & $\begin{array}{l}\text { "Reconociendo la importancia creciente del pensamiento de Zygmunt Bauman, (...) } \\
\text { esta tesis pretende concretar para el campo específico de la educación una compi- } \\
\text { lación que permita, en calidad de herramienta e instrumento de lectura, un abor- } \\
\text { daje con mayor seguridad y luz en el proceso (...). Se aplicará la sistematización } \\
\text { lograda a la realidad educativo-comunicacional de la provincia de San Juan con } \\
\text { el fin de verificar sus posibilidades como herramienta investigativa ajustando los } \\
\text { aspectos que lo permitan y dejando constancia de las limitaciones o problemas } \\
\text { que se presenten, bases posibles para futuras investigaciones". }\end{array}$ \\
\hline
\end{tabular}

Aparecen en el corpus un total de 61 justificaciones, las que se distribuyen según el detalle de la tabla 5 y del gráfico 2 (cfr. tabla 5 y gráfico 2).

Como se advierte, la categoría más frecuente es la justificación "basada en el aporte de los hallazgos", seguida por "basada en la importancia del tema”. Las diferencias sólo alcanzan sig- nificatividad estadística cuando se compara la primera categoría con los dos porcentajes que representan una sola elección ( $z=2,31)$.

\subsection{Citación y referenciación}

Se halla un total de 1.474 citas, con un rango entre 14 y 137, esto es, una gran variabilidad 


\section{TABLA 5}

Frecuencia y porcentaje de tipos de justificación

\begin{tabular}{l|c|c}
\hline \multicolumn{1}{c|}{ Tipo de justificación } & Frecuencia & Porcentaje \\
\hline $\begin{array}{l}\text { Falta genérica de } \\
\text { conocimiento }\end{array}$ & 8 & $13 \%$ \\
\hline $\begin{array}{l}\text { Falta de conocimiento } \\
\text { práctico }\end{array}$ & 5 & $8 \%$ \\
\hline $\begin{array}{l}\text { Basada en la } \\
\text { importancia del tema }\end{array}$ & 15 & $25 \%$ \\
\hline $\begin{array}{l}\text { Basada en los aportes } \\
\text { de los hallazgos }\end{array}$ & 23 & $38 \%$ \\
\hline $\begin{array}{l}\text { Basada en un vacío } \\
\text { metodológico }\end{array}$ & 1 & $1,5 \%$ \\
\hline $\begin{array}{l}\text { Basada en la solución } \\
\text { de un problema }\end{array}$ & 1 & $13 \%$ \\
\hline $\begin{array}{l}\text { Basada en la } \\
\text { corroboración } \\
\text { de una teoría }\end{array}$ & 61 & $100 \%$ \\
\hline
\end{tabular}

(D. S. = 20,10; amplitud intercuartil = 30), con una media de 47,52, una mediana de 43 y un índice de citas por cantidad de palabras de $5,6^{8}$.

El límite inferior del rango corresponde a un proyecto relativamente corto (5.985 palabras) en tanto se ubica en el percentil 25 de la distribución según el número de palabras. La puntuación máxima remite al más largo del corpus (22.313 palabras), que desarrolla la propuesta de una tesis de autor, al que cita repetidamente cuando introduce los textos que estudiará, y que, por otra parte, recurre a varios núcleos temáticos de distintas disciplinas (sociología, pedagogía, ciencias de la comunicación, ética) para caracterizar el objeto del análisis -la educomunicación-.

Respecto de la categorización de las citas, véanse los resultados en la tabla 6 y en el gráfico 3

\section{GRÁFICO 2}

Distribución porcentual de los tipos de justificación

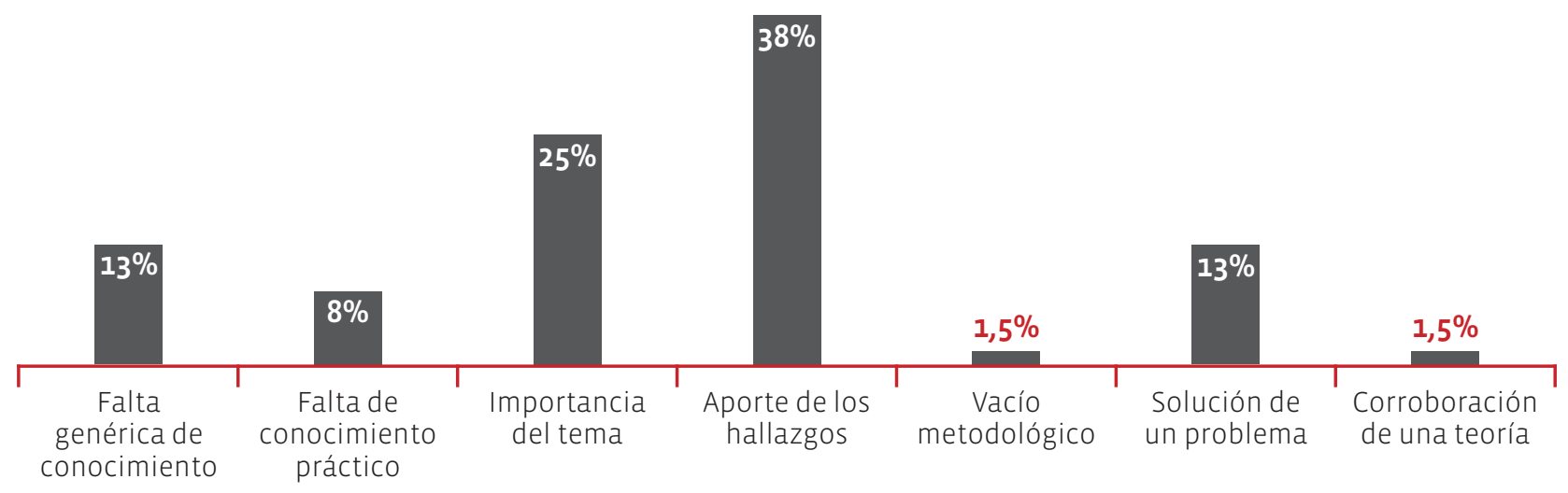

\section{TABLA 6}

Frecuencia y porcentaje de los tipos de citas

\begin{tabular}{c|c|c|c|c|c}
\cline { 2 - 5 } & $\begin{array}{c}\text { Integrada } \\
- \text { literal }\end{array}$ & $\begin{array}{c}\text { Integrada } \\
- \text { no literal }\end{array}$ & $\begin{array}{c}\text { No integrada } \\
- \text { literal }\end{array}$ & $\begin{array}{c}\text { No integrada } \\
- \text { no literal }\end{array}$ & Total \\
\hline Frecuencia & 171 & 662 & 258 & 383 & 1.474 \\
\hline Porcentaje & $11,5 \%$ & $45 \%$ & $17,5 \%$ & $26 \%$ & $100 \%$ \\
\hline
\end{tabular}

8 Para el cálculo del índice de citas, se siguió el procedimiento de dividir el número de citas por la cantidad de palabras y multiplicar el resultado por 1.000 (Sabaj Meruane y Páez Muñoz, 2011: 124). 


\section{GRÁFICO 3}

Distribución porcentual por tipo de cita

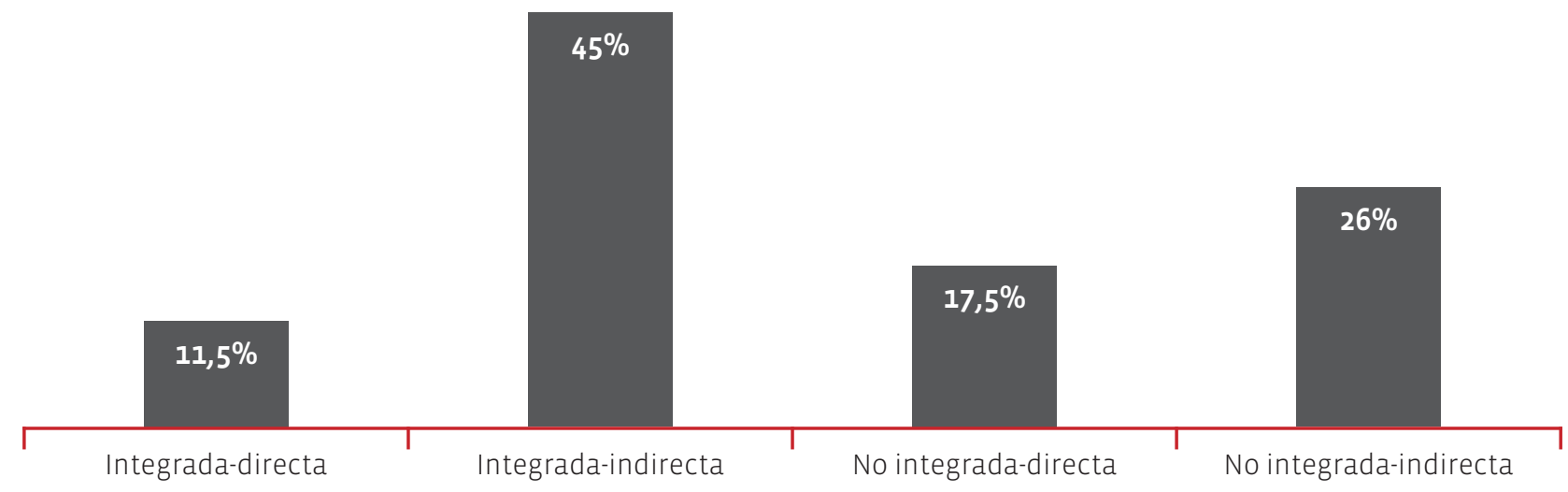

Es claro que priman las citas no literales o indirectas (71\%). La categoría con mayor frecuencia es la integrada indirecta; esta diferencia es estadísticamente significativa $\left(\mathrm{Chi}^{2}=26,037 ; \mathrm{g}\right)=$
3; $p=0,000)$.

En relación con las funciones retóricas de las citas, en la siguiente tabla se muestran ejemplos extraídos del corpus:

\section{TABLA 7}

Ejemplificación de las funciones retóricas de las citas

\begin{tabular}{|c|c|}
\hline Función retórica & Ejemplo \\
\hline Atribución & $\begin{array}{l}\text { "Halliday y Martin (1989) sostienen que la escritura en situación escolar debe acentuar la } \\
\text { relación entre uso del lenguaje y propósito social de los textos". }\end{array}$ \\
\hline Ejemplificación & $\begin{array}{l}\text { "Numerosas y variadas investigaciones han examinado las relaciones entre los } \\
\text { administradores escolares y los profesores. Parailustraresto, (...) Anderson (1991) investigó } \\
\text { cómo los directores manipulaban al profesorado a través de políticas cognitivas y del } \\
\text { lenguaje (...)". }\end{array}$ \\
\hline Referencia & $\begin{array}{l}\text { "A quienes objetan que el niño pequeño no razona, Lipman responde: '(...) se puede decir } \\
\text { que los niños piensan inductiva y deductivamente mucho antes de comenzar a usar el } \\
\text { lenguaje. (...) Más aún, el niño pequeño es tan persistente en ello que, en comparación con } \\
\text { la falta de curiosidad que caracteriza a los adultos, estamos tentados a afirmar que la } \\
\text { conducta filosófica del individuo va disminuyendo con el incremento de la edad.' (Lipman, } \\
\text { 1992: 58-60); cfr., también, Murris (2000) y Parra Molina (2006)". }\end{array}$ \\
\hline Declaración de uso & $\begin{array}{l}\text { "(...) de los instrumentos que evalúan la PTF [Perspectiva de Tiempo Futuro], hemos } \\
\text { seleccionado el Inventario de Perspectiva Temporal de Zimbardo y Boyd (1999), medida } \\
\text { multidimensional de la orientación temporal que incluye la evaluación positiva y negativa } \\
\text { del pasado y del presente, así como la evaluación de la orientación futura”. }\end{array}$ \\
\hline Aplicación & $\begin{array}{l}\text { "La metodología por usar es el dualismo cualitativo - cuantitativo. Para ello, se presentan } \\
\text { las estrategias expuestas por Morgan (1997) para argumentar los motivos principales por } \\
\text { los que he optado por la integración de los dos métodos en el diseño de investigación: (...)". }\end{array}$ \\
\hline Evaluación & $\begin{array}{l}\text { "La autora propone una nueva perspectiva, una mirada profunda, totalizadora y compleja, } \\
\text { a partir del abordaje de la crisis paradigmática, del análisis recursivo de los procesos de } \\
\text { enseñanza-aprendizaje, del concepto de 'sistema abierto'”. }\end{array}$ \\
\hline
\end{tabular}




\begin{tabular}{|c|c|}
\hline Enlace entre fuentes & $\begin{array}{l}\text { "En la didáctica de las ciencias experimentales, las dinámicas de reflexión ayudan a los } \\
\text { alumnos en el aprendizaje de las ciencias (Zee y Roberts, 2002) y al profesor en el contenido } \\
\text { y mejoramiento de su práctica (Pacca y Villena, 2000), dando oportunidad de climas para } \\
\text { el diálogo (Villeni y Franzoni, 2001). Una enseñanza que busca aculturación científica, en } \\
\text { contraposición a la acumulación de contenidos científicos (Matheus, 1994), debe inducir } \\
\text { a los estudiantes a criticar su contenido conceptual para el proceso de construcción } \\
\text { (Carvahlo, 2004). Se trata de tomar como referente una educación crítica de las ciencias } \\
\text { con la que podamos identificarnos (Jiménez y Wanda, 2004)". }\end{array}$ \\
\hline Concurrencia & $\begin{array}{l}\text { “(...) las concepciones sobre el aprendizaje, entendidas como teorías implícitas, orientan } \\
\text { el accionar de los sujetos a la hora de aprender (Scheuer y Pozo, 2006; Atkinson y Claxton, } \\
\text { 2000; Pozo y Scheuer 1999; Pozo et al., 1998; Rodrigo, Rodríguez y Marrero, 1996)". }\end{array}$ \\
\hline Autocita & $\begin{array}{l}\text { "Asimismo, resulta útil nuestro trabajo (Suriani, 2008) que indaga, desde presupuestos } \\
\text { teóricos vigentes de la Sociolingüística, la problemática del tratamiento de la variación } \\
\text { como propiedad inherente a todas las lenguas, que cristaliza la estructura social”. }\end{array}$ \\
\hline
\end{tabular}

A continuación, se presenta la frecuencia absoluta y el porcentaje de cada una:

\section{TABLA 8}

Frecuencia y porcentaje de las funciones retóricas de las citas

\begin{tabular}{|c|c|c|}
\hline Categorías & Frecuencia & Porcentaje \\
\hline Atribución & 859 & $61 \%$ \\
\hline Ejemplificación & 77 & $5,5 \%$ \\
\hline Referencia & 6 & $0,4 \%$ \\
\hline Declaración de uso & 37 & $2,6 \%$ \\
\hline Aplicación & 111 & $7,9 \%$ \\
\hline Evaluación & 119 & $8,5 \%$ \\
\hline Enlace entre fuentes & 116 & $8,3 \%$ \\
\hline Concurrencia & 69 & $4,9 \%$ \\
\hline Autocita & 12 & $0,9 \%$ \\
\hline Total & 1.406 & $100 \%$ \\
\hline
\end{tabular}

La diferencia de totales (1.474 en la primera categorización vs. 1.406 en ésta) obedece a que en aquélla se computa una cita cada vez que se consigna el nombre del autor o la referencialidad; en cambio, en la segunda, de modo análogo a Thompson (2001: 108) y a Petrić (2007: 246), se cuenta una sola cita múltiple en las que aparecen varios autores, como ocurre en Enlace entre fuentes y Concurrencia, e incluso a veces en otras funciones retóricas.

Según se advierte, es muy clara la diferencia a favor de la función atributiva y, obviamente, es una diferencia con significación estadística ya a partir de su comparación con la segunda función en frecuencia -evaluación- $(z=2,75)$. En algunos proyectos, esta preeminencia se traduce en una escritura reproductiva, una suerte de ventrílocuo - una de las categorías de Alves (1992) respecto de los tipos de revisión bibliográfica por evitar - porque el autor sólo habla por boca de otros, ya sea citándolos literalmente, ya sea parafraseando sus ideas. Este estilo es fácilmente reconocible: los párrafos se suceden alternando expresiones "Para Fulano", "Según Sultano", "Como afirma Perengano", "Como Sultano observa", hasta agotar el correspondiente conjunto de verbos sinónimos o de significado bastante cercano - decir, expresar, afirmar, postular, manifestar, opinar, mostrar, explicar, etc.-

Finalmente, se calculó la cantidad de publicaciones incluidas en la sección Referencias o Bibliografía (cfr. gráfico 4). El rango oscila entre 20 y 186 (una evidente variabilidad: D. S. = 38,55; amplitud intercuartil =37), con una media de 60,42 y una mediana de 51

Tal como muestra el gráfico 4, las frecuencias más altas corresponden a las propuestas con menor número de publicaciones, mientras 


\section{GRÁFICO 4}

Frecuencia absoluta del número de referencias bibliográficas por categorías empíricas de cantidad total de publicaciones

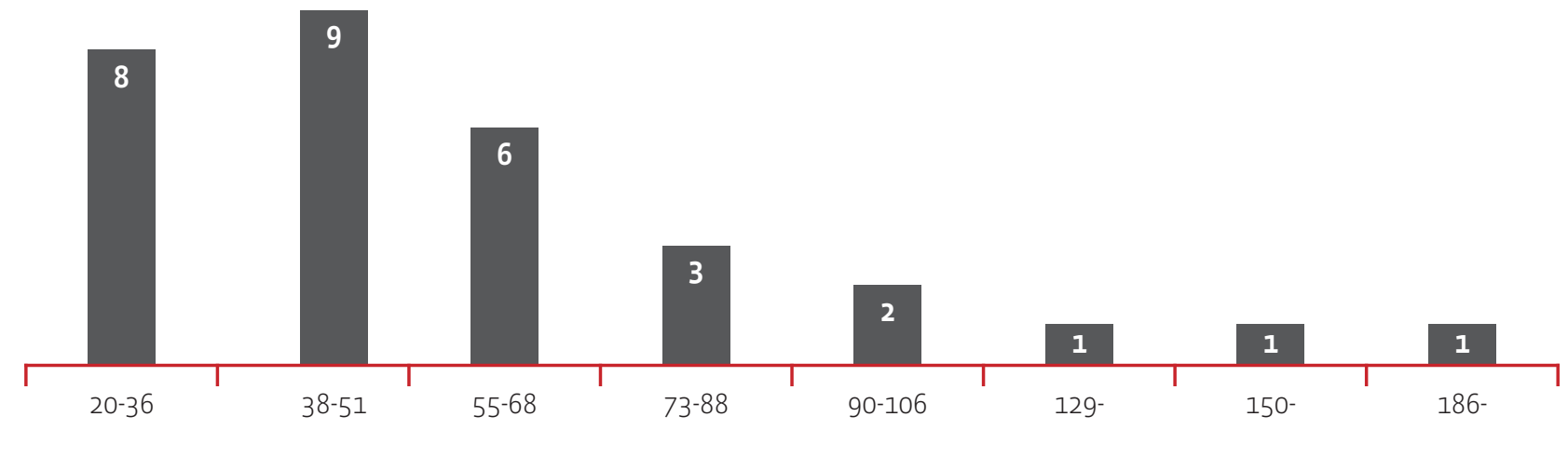

que son tres los proyectos que presentan valores extremos.

Por tipo de publicación (libro, capítulo de libro, artículo científico y otros - ponencia en evento científico, tesis, diccionario y páginas web-), priman los primeros (cfr. gráfico 5) y esta diferencia es estadísticamente significativa ya desde la comparación con los artículos, el segundo tipo más frecuente $(z=5,24)$.

\section{GRÁFICO 5}

Distribución porcentual de tipos de referencias bibliográficas

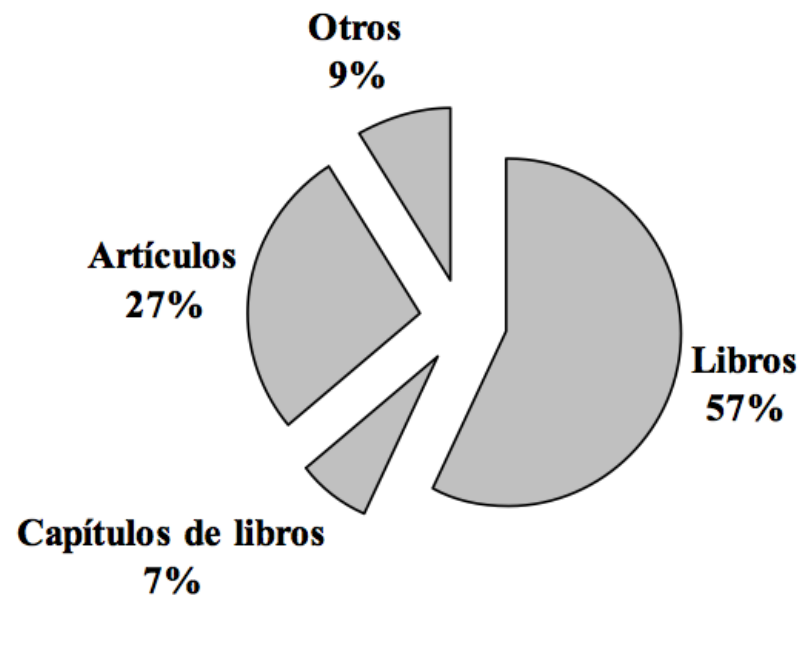

Si bien no era nuestra intención examinar los tipos de referencias bibliográficas según área disciplinar, se pudo constatar que las propuestas enmarcadas en la psicología educacional y en la didáctica incluyen un porcentaje considerable de artículos de revistas científicas (entre 33\% y $70 \%$ ); en cambio, en otras disciplinas (como pedagogía, filosofía de la educación y política educativa) o no se incluye artículo alguno o el índice no supera el $13 \%$. Es claro, entonces, que las ciencias pedagógicas varían en sus modos de comunicación de los resultados: mientras que para algunas las revistas profesionales son un medio propicio, para otras los libros continúan siendo el recurso dominante.

\section{Conclusiones}

Al evaluar un proyecto, el comité académico de un programa de doctorado busca respuesta a tres interrogantes básicos (Wainerman, 1998): 1) ¿qué aprenderemos como resultado de la propuesta de investigación que no sabemos hoy?, 2) ¿por qué vale la pena saberlo?, 3) ¿cómo se garantiza que las conclusiones serán válidas, es decir, fundadas desde el punto de vista científico? Las respuestas, respectivamente, refieren a los antecedentes y formulación del problema, a la justificación y a la metodología. En el presente trabajo, hemos intentado delinear algunos elementos vinculados con las dos primeras — según se concretan en el título, la citación y referenciación, por un lado, y en las razones que justifican el problema seleccionado, por el otro- mediante la aplicación de encuadres teóricos y procedimientos analíticos probados a un corpus de proyectos de formalidad pedagógica.

Si bien su tamaño no habilita generalización 
alguna, ha quedado de manifiesto que las clases de título configuran un conjunto restringido en el que prevalece la construcción nominal que anuncia el tema, categoría en la que, a su vez, distinguiéramos subclases según la modalidad de la investigación: comparativa o correlacional, explicativa y de tesis de autor. Es claro que, por el nivel académico de destino, ninguna propuesta se circunscribe al plano meramente descriptivo y que, si bien dicha construcción nominal se corresponde con la naturaleza prototípica de la ciencia que tiende a clasificar sus objetos de estudio (Soler, 2007), a fin de satisfacer las necesidades de investigadores en formación un contenido por incluir en algún curso del doctorado es la reflexión sobre las implicancias semánticas y pragmáticas del título de una investigación doctoral.

En segundo lugar, priman las citas no literales y la categoría con mayor frecuencia (una diferencia estadísticamente significativa) es la integrada indirecta. Ello parece indicar que el doctorando, un escritor por lo general inexperto, necesita poner el acento en el enunciador autorizado como garantía de relevancia de su trabajo (a veces, este énfasis hace que no logre llevar el hilo conductor del discurso), aunque le basta la paráfrasis, la síntesis, el comentario, la referencia, porque en el área educativa, una vez establecida la opción paradigmática - con la consecuente delimitación de lo que es importante, legítimo y razonable-, se subraya el "acuerdo con las ideas (...) por lo que un análisis pormenorizado de las palabras que utiliza el autor para transmitir esas ideas se hace irrelevante" (Sabaj Meruane y Páez Muñoz, 2011: 126). Además, se halla que el índice de citas por cantidad de palabras es de 5,6, un coeficiente muy cercano al que estos investigadores encuentran en artículos correspondientes a terapia psicológica, disciplina que - como las ciencias de la educación- se ocupa del ámbito del obrar.

Respecto de las funciones retóricas, aunque cada tipo de citación tiene su lugar en el discur- so académico, algunas de dichas funciones se destacan por su contribución a la fuerza del texto. La evaluación, por ejemplo, guarda un vínculo estrecho con la argumentación, en lugar de conformarse con hacer un uso enciclopédico de las publicaciones referidas, mientras que otras funciones - como la atribución o la referencia- están más cercanas a la exposición (Sánchez, 2013). En nuestro corpus prevalece el estilo expositivo en tanto representa el $61 \%$ del total de citas, Io que si bien es esperable en quien se inicia en la investigación - los escritores no expertos, señala Petrić (2007: 251), tratan de demostrar su conocimiento sobre el tema-, plantea la necesidad de incluir la enseñanza de las diferentes funciones retóricas de la citación para enriquecer el discurso científico. Dado que la referencia a literatura sustantiva es parte esencial de la escritura académica y es fuente de dificultades considerables para el escritor novel (Thompson y Tribble, 2001), en algún espacio curricular del plan de estudios del doctorado se debería promover la formación sistemática en cómo analizar, sintetizar y evaluar el conocimiento acumulado.

Finalmente, la justificación del problema aparece como un área débil, poco desarrollada (en este sentido, el 32\% de la muestra o no proporciona razón alguna o anticipa un solo argumento), si se subraya que un proyecto de investigación debe presentar un producto defendible, una exposición razonada y convincente de las razones que lo avalan, a fin de persuadir a un comité académico de su valía.

Si bien el objetivo propio de toda investigación es promover el avance científico, este propósito se puede particularizar en el siguiente agrupamiento ad hoc de los siete tipos de justificación de la importancia de un problema que distinguen Sabaj Meruane y Landea Balin (2012), según que el trabajo:

- Busque completar: 1) la falta genérica de conocimiento, 2) la falta de conocimiento práctico, 3) un vacío metodológico. 
- Se respalde en: 4) la importancia del tema, 5) el aporte de los hallazgos potenciales.

- Se encamine a: 6) la solución de un problema práctico, 7) la corroboración de una teoría.

De esta categorización, en nuestro corpus predomina el segundo grupo - respaldo en la importancia del tema y en el aporte de los haIlazgos, en ese orden-, el que en forma conjunta representa el $63 \%$ de las justificaciones. Por otra parte, como en dichos autores, las menos frecuentes (con una elección cada una) son la basada en el vacío metodológico y en la corroboración empírica de una teoría.

Esta limitada "potencia retórica" (Sabaj Meruane y Landea Balin, 2012) de las razones que avalan un estudio, en el caso de nuestros doctorandos parece explicarse por un rasgo compartido: la mayoría de los candidatos son docentes que deben recorrer el pasaje de una manera normativa de pensar la educación -el objeto de estudio ya está justificado- a una analítica y de una relación muy personal con el fenómeno educativo (la docencia como una labor con un fuerte compromiso afectivo) a una perspectiva universal.

Por otra parte, tanto dicha justificación poco elaborada de la propuesta como la preeminencia de la función atributiva de la citación muestran la necesidad de que el doctorando comience a transitar el paso desde cursante de seminarios, esto es, un consumidor del conocimiento, al rol de investigador, a productor de conocimiento con frecuencia a través de procesos inciertos.

En suma, nuestros resultados, por un lado, pueden constituir un punto de partida para investigar corpus más amplios y para implementar acciones pedagógicas; por otro, respecto de la dinámica del recurso a la literatura científica, plantean dos líneas de interés por profundizar.

La primera, como en el trabajo de Petrić (2007), es el estudio de la relación entre estrategias de citación y calidad del texto, ya que -como es de esperar- los mejores proyectos manifiestan una mayor frecuencia de citas más complejas (evaluación, enlace entre fuentes, concurrencia).

Una segunda línea, que ciertamente requiere la contribución del experto en cada tema, es ponderar el nivel académico de las referencias (por ejemplo, revistas especializadas sobre revistas generales), su actualidad y la incorporación efectiva de las lecturas indispensables (por ejemplo, aquellos textos seminales y últimos respecto de su problema de investigación que no pueden faltar).

\section{Bibliografía citada}

Alves, Alda, 1992: "A 'revisao da bibliografia' em teses e dissertaçoes: Meus tipos inesquecíveis", Cadernos de Pesquisa 81, 53-60.

Beke, Rebecca, 2008: "El discurso académico: la atribución del conocimiento en la investigación educativa", Núcleo 25, 13-35.

Bermúdez, Nicolás, 2008: "Propuestas para un abordaje multidimensional de los géneros en la comunidad discursiva de posgrado", ponencia presentada en el $11 .{ }^{\circ}$ Congreso de la Sociedad Argentina de Lingüística.

Bertely Busquets, María, 2000: Conociendo nuestras escuelas. Un acercamiento etnográfico a la cultura escolar, Barcelona: Paidós.

Boote, David y Penny Belle, 2005: "Scholars Before Researchers: On the Centrality of the Dissertation Literature Review in Research Preparation", Educational Researcher 34, 6, 3-15.

Carlino, Paula, 2005: "La experiencia de escribir una tesis: contextos que la vuelven más difícil", Anales del Instituto de Lingüística XXIV, 41-62.

Crosby, Harry, 1976: "Titles, A Treatise On ...", College Composition \& Communication 27, 4, 387-391.

D'ANDREA, Livia, 2002: "Obstacles to Completion of the Doctoral Degree in Colleges of Education", Educational Research Quarterly 25, 3, 42-58. 
DAY, Robert, 1990: Cómo escribir y publicar trabajos científicos, Washington: Organización Panamericana de Salud.

Difabio de Anglat, Hilda, 2005: Competencias para la comprensión de textos y el pensamiento crítico en nivel medio y universitario. Tesis de doctorado, Universidad Nacional de Cuyo, Mendoza, Argentina.

Difabio de Anglat, Hilda, 2011: "Las funciones del tutor de la tesis doctoral en Educación", Revista Mexicana de Investigación Educativa XVI, 50, 935-959.

Dúo de Brottier, Ofelia, 2005: "La ponencia y el resumen de ponencia" en Liliana Cubo de Severino (coord.): Los textos de la ciencia. Principales clases del discurso académico-científico, Córdoba, Argentina: Comunicarte, 113-152.

Glaser, Barney y Anselm Strauss, 1967: The Discovery of Grounded Theory. Strategies for Qualitative Research, New York: Aldine Publishing Company.

HaRtLey, James, 2007: "There's more to the title than meets the eye: exploring the possibilities", Journal of Technical Writing \& Communication 37, 95-101.

Hartley, James, 2008: Academic Writing and Publishing. A practical handbook, London: Routledge.

JaRA, Eugenio, 1999: "La selección del título en el artículo científico", Revista Cubana de Medicina General Integral 15, 3, 342-345.

Jakobs, Eva-Maria, 2003: "Reproductive writing writing from sources", Journal of Pragmatics 35, 893-906.

Massı, Ma. Palmira, 2005: "Las citas en la comunicación académica escrita”, Revista Iberoamericana de Educación [http://www.rieoei.org/deloslectores, fecha de consulta: 13 de junio de 2008].

Paredes de Meaños, Zulema, 1997: El proyecto institucional en el marco de las transformaciones educativas, Buenos Aires: El Ateneo.

Parodi, Giovanni, 2005: Discurso especializado e instituciones formadoras, Valparaíso: Ediciones Universitarias de Valparaíso.

Petrić, Bojana, 2007: "Rhetorical functions of citations in high and low-rated master's thesis", Journal of English for Academic Purposes 6, 238-253.

PIAGET, Jean, 1984 [1978]: Investigaciones sobre la generalización, Puebla: Premia Editora.

Reyes, Graciela, 1999: Cómo escribir bien en español. Manual de redacción, segunda edición, Madrid: Arco Libros.

Romera Iruela, Ma. Jesús, 1996: "Citas y Referencias Bibliográficas en el Sistema de Comunicación Científica”, Revista Complutense de Educación 7, 1, 243-270.

Sabaj Meruane, Omar y Denisse Landea Balin, 2012: "Descripción de las formas de justificación de los objetivos en artículos de investigación en español de seis áreas científicas”, Onomázein 25, 1, 315-344.

Sabaj Meruane, Omar y Dennis Paez Muñoz, 2011: "Tipos y funciones de las citas en artículos de investigación de tres disciplinas", Literatura y Lingüística 22, 117-134.

Saltalamacchia, Homero, 2005: Del proyecto al análisis: aportes a una investigación cualitativa socialmente útil, tomo I, Buenos Aires: El Artesano.

Sánchez, David, 2011: Las funciones retóricas de la citación en la escritura académica universitaria. Estudio comparado del género de memorias de máster en nativos españoles y estudiantes filipinos de ELE. Tesis para el Diploma de Estudios Avanzados (D. E. A.) en Lingüística Aplicada a la Enseñanza del Español como Lengua Extranjera, Universidad Nebrija, España.

Sanchez, David, 2012: "El uso de las funciones de las citas en la estructura retórica de las Introducciones de memorias de máster escritas en 
español por estudiantes nativos españoles y no nativos filipinos", Revista Nebrija de Lingüística Aplicada 12, 6, 137-172.

Sánchez, David, 2013: "Aplicabilidad de la tipología de funciones retóricas de las citas al género de la memoria de máster en un contexto transcultural de enseñanza universitaria", Revista Signos. Estudios de Lingüística 46, 81, 82-104.

Soler, Viviana, 2007: "Writing titles in science: An exploratory study", English for Specific Purposes 26, 90-102.

Swales, John, 1990: Genre Analysis. English in Academic and Research Settings, Cambridge: Cambridge University Press.

Thompson, Paul, 2001: A pedagogically-motivated corpus-based examination of PhD theses: macroestructure, citation practices and uses of modal verbs. PhD thesis, University of Reading.

Thompson, Paul y Chris Tribble, 2001: "Looking at citations: Using corpora in English for academic purposes", Language Learning and Technology 5, 3, 91-105.

Van DIJk, Teun y Walter KInTSCH, 1983: Strategies of Discourse Comprehension, Orlando: Academic Press.

Wainerman, Catalina, 1998: "Formulación de proyectos" en Catalina WaInerman, Alicia Gorri y Daniel Prieto Castillo: Pilares de la investigación. Formulación. Evaluación. Comunicación, Mendoza: Ediunc, 13-36. 